Published on Web 07/10/2004

\title{
Functional Vibrational Spectroscopy of a Cytochrome $c$ Monolayer: SEIDAS Probes the Interaction with Different Surface-Modified Electrodes
}

\author{
Kenichi Ataka and Joachim Heberle* \\ Contribution from the Forschungszentrum Jülich, IBI-2: Structural Biology, \\ 52425 Jülich, Germany
}

Received March 23, 2004; E-mail: j.heberle@fz-juelich.de

\begin{abstract}
Electrochemically induced infrared difference spectra of cytochrome $c$ on various chemically modified electrodes (CMEs) are recorded by exploiting the surface-enhancement exerted by a granular gold film. We have recently developed surface-enhanced infrared difference absorption spectroscopy (SEIDAS), which provides acute sensitivity to observe the minute enzymatic change of a protein on the level of a monolayer. By these means, we demonstrate that the relative band intensities in the potentialinduced difference spectra of adsorbed cytochrome $c$ are significantly dependent on the type of CME used (mercaptopropionic acid, mercaptoethanol, 4,4'-dithiodipyridine, or L-cysteine). These differences are attributed to the altered interaction of cytochrome $c$ with the headgroup of the various CMEs leading to variations in surface orientation and relative distance from the surface. Nevertheless, the peak positions of the observed bands are identical among the CMEs employed. This implies that the internal conformational changes induced by the redox reaction of the adsorbed cytochrome $c$ are not disturbed by the interaction with the CME and that full functionality of the protein is retained. Finally, we critically discuss our results within the framework of the different models for cytochrome $c$ adsorption on CMEs.
\end{abstract}

\section{Introduction}

Monolayers of proteins are very attractive for the investigation of the structure and dynamics of intermolecular electron transfer within the native cell. Cytochrome $c$ (cyt $c$ ), a water-soluble $12.5 \mathrm{kDa}$ protein that mediates single-electron transfer between the integral membrane protein complexes of the respiratory chain, is regarded as the model system for studying details of the electron-transfer pathway, the role of the protein in setting the redox potential, molecular recognition of the redox partners, etc. ${ }^{1}$

A very powerful method for functional studies of this protein is cyclic voltammetry. Enormous contributions have been made with this methodology to the understanding of the thermodynamics and kinetics of cyt $c^{1}$ as well as to the development of bioelectronics. ${ }^{2,3}$ Although voltammetry of the cyt $c$ redox reaction at a bare metal electrode surface has been successfully demonstrated by careful control of the purity of the cyt $c$ preparation and the solution conditions, ${ }^{4}$ chemically modified electrodes (CMEs) are more conventionally employed due to the ease of preparation and the prevention of passivation. Initially, pyridine-terminated self-assembled monolayers (SAMs)

* Corresponding author. Phone: (+49) 2461-61-2024. Fax: (+49) 246161-2020.

(1) Fedurco, M. Coord. Chem. Rev. 2000, 209, 263-331.

(2) Page, C. C.; Moser, C. C.; Chen, X.; Dutton, P. L. Nature 1999, 402, 4752.

(3) Willner, I.; Katz, E. Angew. Chem., Int. Ed. 2000, 39, 1180-1218.

(4) Reed, D. E.; Hawkridge, F. M. Anal. Chem. 1987, 59, 2334-2339.

10.1021/ja048346n CCC: $\$ 27.50$ @ 2004 American Chemical Society were used such as $4,4^{\prime}$-bipyridine ${ }^{5}$ or dithiodipyridine, ${ }^{6}$ but soon other types of CMEs were employed, including hydroxyl- ${ }^{7}$ and carboxyl-terminated alkanethiols. ${ }^{8-10}$ Despite the recent development of a variety of CMEs, the chemical properties of each CME have different influences on the reaction of cyt $c$ and eventually complicate the understanding of the system. The deficiency of the electrochemical methods for obtaining structural information has limited the understanding of the correlation between the interfacial protein structure and the reaction kinetics.

This gap can be filled by linking the electrochemical method with spectroscopic detection. Niki and co-workers have applied $\mathrm{UV} / \mathrm{vis}$ reflectance spectroscopy to determine the electrontransfer rate constant of cyt $c .{ }^{11-14}$ This method is advantageous regarding the sensitivity and ability to detect rapid ET reaction. Fluorescence spectroscopy has been used to determine the

(5) Eddowes, M. J.; Hill, H. A. O. J. Am. Chem. Soc. 1979, 101, 4461-4464.

(6) Taniguchi, I.; Toyosawa, K.; Yamaguchi, H.; Yasukouchi, K. J. Electroanal. Chem. 1982, 140, 187-193.

(7) Terrettaz, S.; Cheng, J.; Miller, C. J. J. Am. Chem. Soc. 1996, 118, 78577858.

(8) Tarlov, M. J.; Bowden, E. F. J. Am. Chem. Soc. 1991, 113, 1847-1849.

(9) Leopold, M. C.; Bowden, E. F. Langmuir 2002, 18, 2239-2245.

(10) Clark, R. A.; Bowden, E. F. Langmuir 1997, 13, 559-565.

(11) Avila, A.; Gregory, B. W.; Niki, K.; Cotton, T. M. J. Phys. Chem. B 2000, 104, 2759-2766.

(12) Feng, Z. Q.; Imabayashi, S.; Kakiuchi, T.; Niki, K. J. Electroanal. Chem. 1995, 394, 149-154.

(13) Feng, Z. Q.; Imabayashi, S.; Kakiuchi, T.; Niki, K. J. Chem. Soc. Faraday Trans. 1997, 93, 1367-1370.

(14) Niki, K.; Hardy, W. R.; Hill, M. G.; Li, H.; Sprinkle, J. R.; Margoliash, E.; Fujita, K.; Tanimura, R.; Nakamura, N.; Ohno, H.; Richards, J. H.; Gray, H. B. J. Phys. Chem. B 2003, 107, 9947-9949. 
surface orientation of the heme chromophore in cyt $c$ on surfaces with various modifications. ${ }^{15-17}$ Other spectroscopic techniques such as surface plasmon resonance and second harmonic generation $^{18,19}$ have been used to determine the adsorption kinetics of cyt $c$ to chemically modified surfaces.

Vibrational spectroscopy is prominent among the various spectroscopic techniques, as it provides chemical information. Surface-enhanced resonance Raman spectroscopy (SERRS) has been extensively applied to the characterization of the heme chromophore of cyt $c .^{20-27}$ Recently, Hildebrandt and coworkers recorded time-resolved SERS spectra of the redox reaction of cyt $c$ and succeeded in relating the reaction kinetics to the structure of the heme moiety of cyt $c .^{26,28}$ Despite these accomplishments, however, the Raman approach suffers from the limitation that only the chromophore is observed due to the resonance condition.

IR spectroscopy provides the vibrational spectrum of the entire protein. The analysis of the amide band is frequently employed for the quantitative determination of the secondary structure of a protein. ${ }^{29-33}$ Since the amide I band comprises a manifold of overlapping bands from specific types of secondary structure, analysis by second derivative or Fourier deconvolution has often been used to separate these characteristic frequencies. Dong et al. ${ }^{34}$ have applied this approach for the analysis of redox-dependent structural changes of cyt $c$ in aqueous solution. Although they observed distinct changes in the amide I vibration at various redox stages of cyt $c$, the structural derivation based on their band assignment is at odds with recent results from NMR $^{35-38}$ and X-ray crystallography. ${ }^{39-41}$ This might be due to the fact that they overestimate the fraction of individual secondary structures by fitting of band areas in second-derivative spectra. Such a procedure may introduce a source of error due to subjectivity and uncertainty in the definition of the baseline. ${ }^{30}$

(15) Edmiston, P. L.; Lee, J. E.; Cheng, S. S.; Saavedra, S. S. J. Am. Chem. Soc. 1997, 119, 560-570.

(16) Lee, J. E.; Saavedra, S. S. Langmuir 1996, 12, 4025-4032.

(17) Tronin, A.; Edwards, A. M.; Wright, W. W.; Vanderkooi, J. M.; Blasie, J. K. Biophys. J. 2002, 82, 996-1003.

(18) Salafsky, J. S.; Eisenthal, K. B. J. Phys. Chem. B 2000, 104, 7752-7755.

(19) Lahiri, J.; Isaacs, L.; Tien, J.; Whitesides, G. M. Anal. Chem. 1999, 71 $777-790$

(20) Macdonald, I. D. G.; Smith, W. E. Langmuir 1996, 12, 706-713.

(21) Walker, D. S.; Hellinga, H. W.; Saavedra, S. S.; Reichert, W. M. J. Phys. Chem. 1993, 97, 10217-10222.

(22) Niaura, G.; Gaigalas, A. K.; Vilker, V. L. J. Electroanal. Chem. 1996, 416, 167-178.

(23) Dick, L. A.; Haes, A. J.; Van Duyne, R. P. J. Phys. Chem. B 2000, 104, $11752-11762$.

(24) Hildebrandt, P.; Stockburger, M. Biochemistry 1989, 28, 6722-6728.

(25) Hildebrandt, P.; Stockburger, M. Biochemistry 1989, 28, 6710-6721.

(26) Hildebrandt, P.; Murgida, D. H. Bioelectrochemistry 2002, 55, 139-143.

(27) Wackerbarth, H.; Hildebrandt, P. Chemphyschem. 2003, 4, 714-724.

(28) Murgida, D. H.; Hildebrandt, P. Angew. Chem., Int. Ed. 2001, 40, 728731.

(29) Krimm, S.; Bandekar, J. Adv. Protein Chem. 1986, 38, 181-364.

(30) Surewicz, W. K.; Mantsch, H. H.; Chapman, D. Biochemistry 1993, 32, 389-394

(31) Venyaminov, S. Y.; Kalnin, N. N. Biopolymers 1990, 30, 1259-1271.

(32) Chirgadze, Y. N.; Nevskaya, N. A. Biopolymers 1976, 15, 607-625.

(33) Kubelka, J.; Keiderling, T. A. J. Am. Chem. Soc. 2001, 123, 12048-12058.

(34) Dong, A.; Huang, P.; Caughey, W. S. Biochemistry 1992, 31, 182-189.

(35) Feng, Y. Q.; Roder, H.; Englander, S. W. Biochemistry 1990, 29, 34943504 .

(36) Qi, P. X.; Distefano, D. L.; Wand, A. J. Biochemistry 1994, 33, 64086417

(37) Lo, T. P.; Guillemette, J. G.; Louie, G. V.; Smith, M.; Brayer, G. D. Biochemistry 1995, 34, 163-171.

(38) Banci, L.; Bertini, I.; Gray, H. B.; Luchinat, C.; Reddig, T.; Rosato, A.; Turano, P. Biochemistry 1997, 36, 9867-9877.

(39) Takano, T.; Dickerson, R. E. J. Mol. Biol. 1981, 153, 79-94.

(40) Takano, T.; Dickerson, R. E. J. Mol. Biol. 1981, 153, 95-115

(41) Bushnell, G. W.; Louie, G. V.; Brayer, G. D. J. Mol. Biol. 1990, 214, $585-595$.
The detection of a single vibration out of the $3 N+6$ degrees of vibrational freedom of a protein is feasible by applying reaction-induced infrared difference spectroscopy. ${ }^{42-47}$ In this approach, functionally relevant vibrational changes are resolved by recording the differences between the resting and active states of the protein. The formation of the active state depends on the enzymatic reaction of the protein, which can be triggered by external stimuli like light, chemicals, or voltage. This approach has been extremely successful in the elucidation of the catalytic mechanism of many biological machines. Moss et al. ${ }^{48}$ were the first to record the electrochemically induced IR difference spectrum of cyt $c$. In a later study, ${ }^{49}$ they elucidated the influence of the chemical nature of the surface modifier on the redox reaction of cyt $c$. However, direct evidence of an interaction between the surface modifier and the adsorbed cyt $c$ cannot be provided by IR spectra of cyt $\mathrm{c}$ residing in the bulk phase. Since such an interaction takes place only between the modifier and the adjacent first layer of protein, the elucidation of the monolayer structure of the adsorbed cyt $c$ is mandatory for understanding the redox process at the electrochemical interfaces.

The in situ detection of a protein monolayer is challenging because conventional IR spectroscopy suffers from poor sensitivity. Due to the finite adsorption area, the surface concentration of the adsorbed protein is limited to a few pmol per $\mathrm{cm}^{2} .{ }^{1}$ Moreover, reaction-induced infrared spectral changes of proteins are usually much less than $1 \%$ of the absolute intensity. The strong background signal from the bulk phase may also conceal the small signal from the monolayers. To overcome these difficulties, we employ surface-enhanced infrared absorption spectroscopy ${ }^{50}$ combined with the difference IR approach, namely, surface enhanced infrared difference absorption spectroscopy (SEIDAS). The acute sensitivity of SEIDAS, which enhances the vibrational absorption of adsorbed molecules by more than 2 orders of magnitude, enables the detection of minute spectral changes of the adsorbed protein. As an optical nearfield effect, only those vibrations are enhanced that arise from molecules that are within a very short distance from the surface $(<8 \mathrm{~nm})$. This property facilitates the elimination of the bulk contribution from the IR spectrum and selectively detects signals from the adsorbed monolayer. ${ }^{51-53}$

In a previous work, we have successfully applied SEIDAS to record redox-induced IR difference spectra from a monolayer of cyt $c$ in situ. ${ }^{54}$ In this work, we employ SEIDAS for the study of the electron-transfer taking place at the interface between adsorbed cyt $c$ and various surface modifiers. The resulting SEIDA spectra exhibit spectral features that directly indicate the presence of different surface orientations and

(42) Mäntele, W. Trends Biochem. Sci. 1993, 18, 197-202.

(43) Braiman, M. S.; Rothschild, K. J. Annu. Rev. Biophys. Biophys. Chem. 1988, 17, 541-570.

(44) Gerwert, K. Curr. Opin. Struct. Biol. 1993, 3, 769-773.

(45) Vogel, R.; Siebert, F. Curr. Opin. Chem. Biol. 2000, 4, 518-523.

(46) Zscherp, C.; Barth, A. Biochemistry 2001, 40, 1875-1883.

(47) Nyquist, R. M.; Ataka, K.; Heberle, J. Chembiochem 2004, 5, 431-436.

(48) Moss, D.; Nabedryk, E.; Breton, J.; Mäntele, W. Eur. J. Biochem. 1990 $187,565-572$

(49) Schlereth, D. D.; Mäntele, W. Biochemistry 1993, 32, 1118-1126.

(50) Osawa, M. Surface-Enhanced Infrared Absorption Spectroscopy. In Handbook of Vibrational Spectroscopy; Chalmers, J. M., Griffiths, P. R., Eds. Wiley: Chichester, 2002; pp 785-799.

(51) Ataka, K.; Osawa, M. Langmuir 1998, 14, 951-959.

(52) Ataka, K.; Osawa, M. J. Electroanal. Chem. 1999, 460, 188-196.

(53) Ataka, K.; Yotsuyanagi, T.; Osawa, M. J. Phys. Chem. 1996, 100, $10664-$ 10672.

(54) Ataka, K.; Heberle, J. J. Am. Chem. Soc. 2003, 125, 4986-4987. 


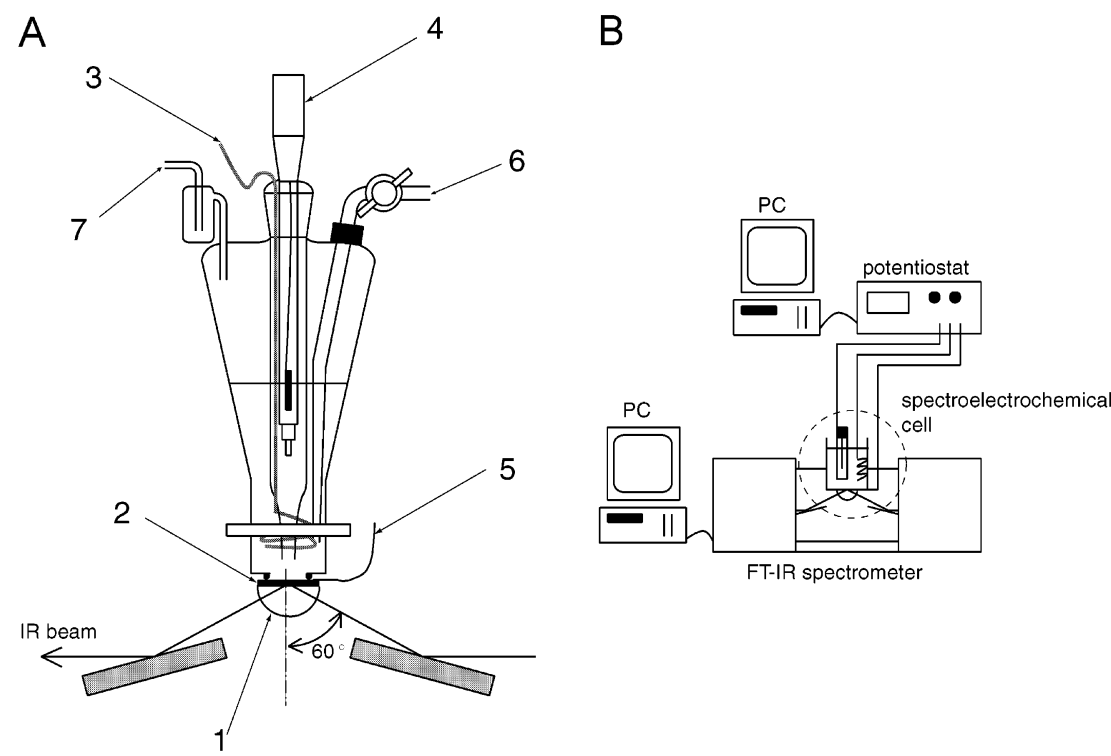

Figure 1. (A) Spectroelectrochemical cell employed for SEIDA spectroscopy, (1) hemicylindric silicon prism, (2) Au thin-film layer, (3) Pt counter electrode, (4) $\mathrm{Ag} / \mathrm{AgCl} / \mathrm{saturated} \mathrm{KCl}$ reference electrode, (5) Cu lead contact to Au thin film, (6) $\mathrm{N}_{2}$ purge gas inlet, (7) $\mathrm{N}_{2}$ purge gas outlet. The body of the cell is made of glass. (B) Schematic drawing of the experimental setup.

structure of the adsorbed cyt $c$ molecules. On this basis, we performed a reassignment of the observed vibrational bands with the help of structural data obtained from NMR spectroscopy ${ }^{35-38}$ and X-ray crystallography. ${ }^{39-41}$

\section{Materials and Methods}

Au Film Preparation. A thin gold film was prepared on the flat surface of a single reflection silicon $(\mathrm{Si})$ hemicylindrical prism $(20 \mathrm{~mm}$ width $\times 25 \mathrm{~mm}$ height, $10 \mathrm{~mm}$ radius) by an electroless (chemical) deposition technique (see the literature ${ }^{55}$ for details). The surface of the Si substrate was polished with aluminum oxide powder ( $1 \mu \mathrm{m}$ size), followed by immersion in a $40 \mathrm{wt} \%$ aqueous solution of $\mathrm{NH}_{4} \mathrm{~F}$ for 3 min. Subsequently, the flat surface of the Si prism was exposed to a 1:1:1 volume mixture of (1) $0.03 \mathrm{M} \mathrm{NaAuCl}_{4}$ (Aldrich), (2) $0.3 \mathrm{M}$ $\mathrm{Na}_{2} \mathrm{SO}_{4}+0.1 \mathrm{M} \mathrm{Na}_{2} \mathrm{~S}_{2} \mathrm{O}_{3}+0.1 \mathrm{M} \mathrm{NH}_{4} \mathrm{Cl}$ (Merck), and (3) $2 \mathrm{vol} \%$ HF (Riedle-de Haen) solution for 60-90 s each. After being rinsed with water, the $\mathrm{Si}$ prism was transferred into a $0.1 \mathrm{M} \mathrm{H}_{2} \mathrm{SO}_{4}$ solution. The gold film surface was cleaned by several oxidation-reduction cycles in the range between 0.1 and $1.4 \mathrm{~V}$ ( $\mathrm{vs} \mathrm{Ag} / \mathrm{AgCl}$ ).

Surface Modification. The surface of the gold film was modified by (electron-transfer) promoter molecules with the following compounds: mercaptopropionic acid (MPA, Aldrich), mercaptoethanol (ME, Sigma), 4,4'-dithiodipyridine (DTDP, Sigma), and L-cysteine (Cys, Sigma). Surface modification was performed by exposing the gold surface for $15 \mathrm{~min}$ to a $1 \mathrm{mM}$ aqueous solution of MPA or ME. For DTDP and Cys, a $10 \mathrm{mM}$ solution was used. The adsorption kinetics of these short-chain thiols saturate very quickly under these conditions (see Figure S1 in Supporting Information). After several rinsing steps with pure water, the modified gold thin film/Si prism was mounted into the spectroelectrochemical cell.

Spectroelectrochemical Cell. The spectroelectrochemical cell is depicted in Figure 1A. The Si prism represents the bottom of the electrochemical cell that is tightened with a Viton O-ring. A Cu plate serves as the electric contact to the gold film. A Pt wire and a Ag/ $\mathrm{AgCl}$ electrode with saturated $\mathrm{KCl}$ solution $(197 \mathrm{mV}$ vs $\mathrm{NHE}$ at 25 ${ }^{\circ} \mathrm{C}$ ) were used as counter and reference electrodes, respectively. An electrolyte solution of $10 \mathrm{mM} \mathrm{K} \mathrm{SO}_{4}$ and $10 \mathrm{mM}$ phosphate buffer $(\mathrm{pH} 7)$ was used in all the experiments. The volume of the electrolyte solution in the cell was about $50 \mathrm{~mL}$. The infrared (IR) beam was

(55) Miyake, H.; Ye, S.; Osawa, M. Electrochem. Commun. 2002, 4, $973-$ 977. coupled into the Si prism at an incident angle of $60^{\circ}$ (ATR-Kretschmann configuration). The totally reflected IR signal from the Si/Au thinfilm/solution interface was detected by a nitrogen-cooled MCT detector of the FT-IR spectrometer (Bruker IFS 66v). All glasswares were cleaned by overnight soaking in a 1:1 mixture of $\mathrm{HNO}_{3} / \mathrm{H}_{2} \mathrm{SO}_{4}$ solution followed by rinsing with ultrapure water $(>18 \mathrm{M} \Omega / \mathrm{cm})$. Horse heart cytochrome $c$ (cyt $c$, Sigma) was used without further purification. All experiments were handled at room temperature.

Kinetic FT-IR Spectroscopy to Monitor the Adsorption of Cytochrome $\boldsymbol{c}$. A reference spectrum of the electrolyte was recorded in the absence of cyt $c$. Subsequently, $1 \mathrm{~mL}$ of $100 \mu \mathrm{M}$ cyt $c$ dissolved in $10 \mathrm{mM}$ buffer solution was added to the cell to achieve a final concentration of $2 \mu \mathrm{M}$ cyt $c$. Adsorption of cyt $c$ took place at the open-circuit potential. The electrolyte solution was constantly stirred by bubbling $\mathrm{N}_{2}$ gas through the gas inlet (6 in Figure 1A) during the measurement. Concomitant with the addition of cyt $c$, sample spectra were acquired at a spectral resolution of $4 \mathrm{~cm}^{-1}$. For each spectrum, 640 scans were averaged, requiring approximately $1 \mathrm{~min}$ for each acquisition. Therefore, the time points indicated in the figures represent the midpoint for each acquisition period.

Potential-Induced FT-IR Difference Spectroscopy under SteadyState Conditions. The redox-induced IR difference spectrum of cyt $c$ in aqueous solution was recorded with an ATR accessory. ${ }^{56}$ Onto the internal reflection element was placed $10 \mu \mathrm{L}$ of an aqueous solution of $4 \mathrm{mM}$ air-oxidized cyt $c$. The recorded IR spectrum served as the reference for the subsequent spectrum, where the cyt $c$ solution is reduced by the addition of a grain of solid sodium dithionite $\left(\mathrm{Na}_{2} \mathrm{~S}_{2} \mathrm{O}_{4}\right)$.

The setup for the potential-induced FT-IR difference experiments of a protein monolayer is depicted in Figure 1B. The electrode potential was controlled by a potentiostat (Autolab PGSTAT 12, Metrohm, Filderstadt, Germany), which allowed for the determination of the electrochemical response of cyt $c$ in contact with the various CMEs by cyclic voltammetry. A single- beam background IR spectrum was taken at $-0.1 \mathrm{~V}$, where the adsorbed cyt $c$ is fully reduced. Then, the potential was increased and a sample spectrum was acquired. This procedure was repeated by stepping successively to more positive potentials. Typically, 640 scans were averaged for each pair of background and sample spectra. The whole procedure was repeated eight times, and difference spectra at the respective electrode potentials

(56) Nyquist, R. M.; Heitbrink, D.; Bolwien, C.; Wells, T. A.; Gennis, R. B.; Heberle, J. FEBS Lett. 2001, 505, 63-67. 


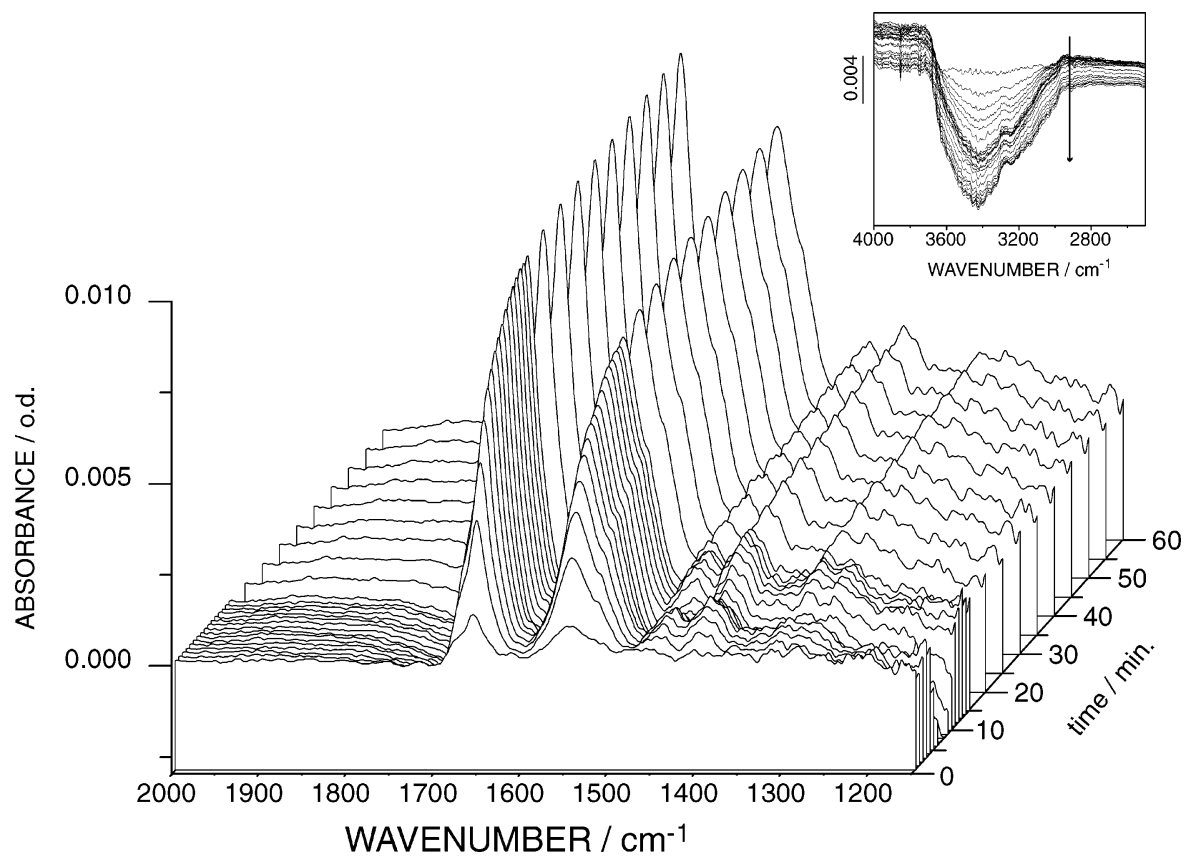

Figure 2. Set of kinetic IR spectra for the adsorption process of cytochrome $c$ on a Au electrode modified with mercaptoethanol SAM. Spectra were recorded each $1 \mathrm{~min}$ for the first $15 \mathrm{~min}$ after addition of cyt $c$. Subsequent spectra were recorded at an interval of $5 \mathrm{~min}$. Inset: $\mathrm{O}-\mathrm{H}$ stretching region of water.

were averaged to improve the signal-to-noise ratio. This procedure ensures the minimization of baseline drifts and other artifacts that may appear during the long measurement time. Therefore, neither baseline correction nor any smoothing procedures needed to be carried out on the observed spectra.

It should be noted that the ATR-Kretschmann configuration provides faster electrochemical response than the conventional thin-layer configuration because the solution resistance is reduced. A chronoamperometric experiment where the voltage was stepped from $-0.2 \mathrm{~V}$ to +0.2 $\mathrm{V}$ showed that the charging current vanishes within $50 \mathrm{~ms}$. This characteristic is advantageous for a short equilibration time of our measurement system.

\section{Results}

Adsorption of Cytochrome $c$ on the Chemically Modified Au Surface. The kinetics of the adsorption of cyt $c$ to a selfassembled monolayer of mercaptoethanol (ME) have been followed by kinetic FT-IR spectroscopy (Figure 2). Two strong positive bands appear that increase in intensities after addition of cyt $c$ to the electrolyte solution. These two major peaks of the adsorbed cyt $c$ are located at 1659 and $1550 \mathrm{~cm}^{-1}$. They are assigned to the amide $\mathrm{I}$ (mainly $\mathrm{C}=\mathrm{O}$ stretching vibration of the peptide bond) and amide II modes (coupled mode of the $\mathrm{NH}$ in-plane bending, $\mathrm{CN}$ stretching, and $\mathrm{C}_{\alpha} \mathrm{C}$ stretching vibration), respectively, of the protein backbone. ${ }^{29}$ The shoulder at around $1680 \mathrm{~cm}^{-1}$ is assigned to the amide I band of a different secondary structural element than that of $1659 \mathrm{~cm}^{-1}$. The lower shoulder of amide II at $1515 \mathrm{~cm}^{-1}$ has been assigned to the phenyl ring stretching vibration of the tyrosine residues. ${ }^{57}$ Overall, the absorption spectrum of cyt $c$ adsorbed to a ME SAM corresponds well to the spectrum of cyt $c$ in bulk solution. ${ }^{34,48}$

Spectral contributions from cyt $c$ molecules in bulk solution can be entirely neglected since their absorbance is less than $10^{-4}$

(57) Wright, W. W.; Laberge, M.; Vanderkooi, J. M. Biochemistry 1997, 36, $14724-14732$.

9448 J. AM. CHEM. SOC. " VOL. 126, NO. 30, 2004 (data not shown). Gravitational accumulation of cyt $c$ on the gold surface is also not plausible since the electrolyte solution is constantly stirred by nitrogen gas bubbling. Concomitant with the appearance and the increase of the bands of adsorbed cyt $c$, broad negative bands appear at the high-frequency edge of the amide I band (Figure 2) and at $3400 \mathrm{~cm}^{-1}$ (insert of Figure 2). These bands are due to the $\mathrm{H}-\mathrm{O}-\mathrm{H}$ bending and the $\mathrm{O}-\mathrm{H}$ stretching mode, respectively, of the water molecules that are expelled from the gold surface during the adsorption of cyt $c .^{52}$

Despite the similarity of the IR absorption spectra of cyt $c$ in solution and adsorbed to ME SAM, the chemical properties of the modified surface critically affect the adsorption kinetics. Figure 3 shows the kinetics of adsorption by plotting the normalized absorbance of the amide I band versus time. It is evident that in the presence of the DTDP or the Cys-modified gold surface, $90 \%$ of the monolayer adsorption is completed within 1 min after addition of cyt $c$. In contrast, the adsorption on the ME and MPA surfaces is rather sluggish. Although about $80 \%$ of the monolayer adsorption is accomplished within the first $15 \mathrm{~min}$, the adsorption still continues up to ca. $30 \mathrm{~min}$ in the case of the MPA-modified surface. It has been suggested that the carboxyl-terminated SAM (MPA) creates the strongest interaction and, therefore, provides the highest coverage of cyt $c .{ }^{1}$ However, a hydroxyl-terminated SAM is suggested to exert the weakest interaction which might even lead to complete failure of adsorption of cyt $c$ (which is contrary to our observation, Figure 3, vide infra). ${ }^{7,58}$ Nevertheless, similar adsorption behavior is observed with MPA and ME, which is in contrast to the fast adsorption kinetics of the weakly interacting SAM formed by DTDP or Cys ${ }^{1}$. Our results provide evidence that the adsorption kinetics is not simply proportional to the strength of the electrostatic interaction and, yet, reveals that a different degree of interaction exists between cyt $c$ and the surface modifiers. 


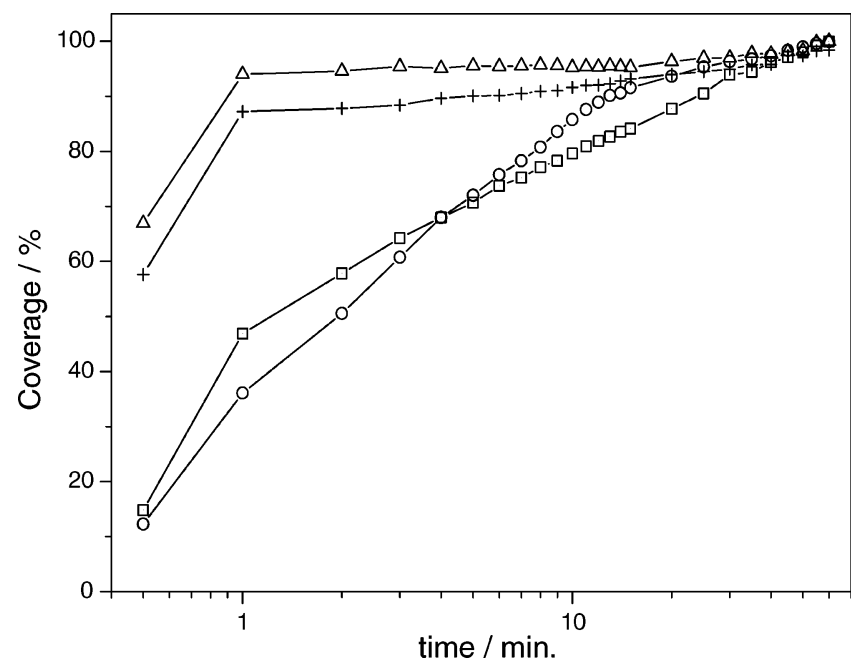

Figure 3. Plot of the surface coverage of cyt $c$ on various CMEs versus time on a logarithmic scale; ( $\square$ ) mercaptopropionic acid (MPA), (O) mercaptoethanol (ME), $(+)$ : cysteine (Cys), $(\triangle)$ dithiodipyridine (DTDP). The surface coverage of cyt $c$ has been derived from the intensity of the amide I band at $1658 \mathrm{~cm}^{-1}$ normalized to the intensity at saturated coverage ( $>60 \mathrm{~min}$ ). Data points are connected by straight lines.

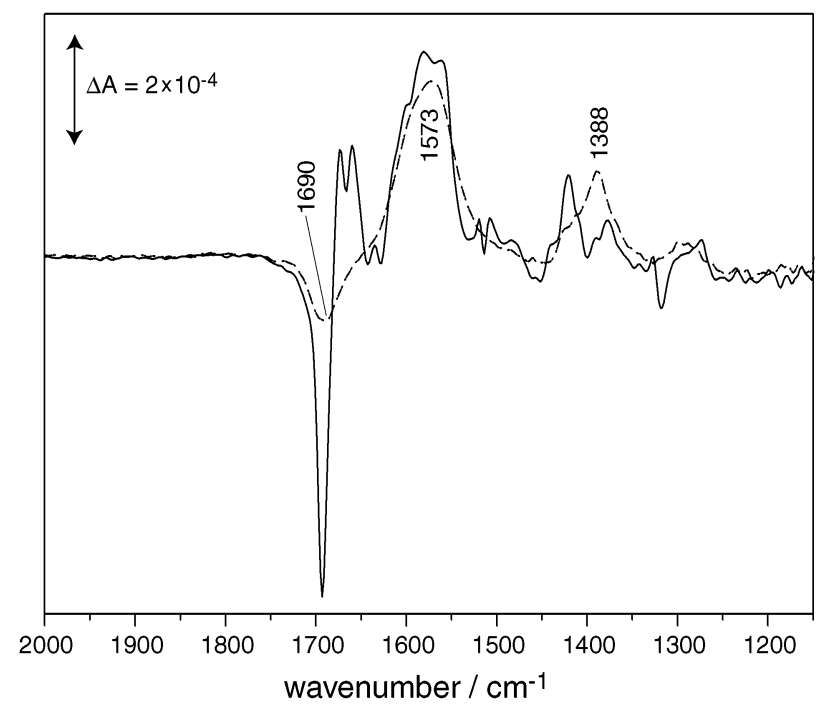

Figure 4. Potential-induced IR difference spectrum of a pure MPA CME (dashed line) and of a monolayer of cyt $c$ adsorbed to the MPA CME (continuous line). Reference and sample spectra were recorded at $-0.1 \mathrm{~V}$ (fully reduced state) and $+0.15 \mathrm{~V}$ (fully oxidized state), respectively.

Potential-Induced IR Difference Spectra of Cytochrome c Adsorbed to a Carboxyl-Terminated SAM. The continuous line in Figure 4 represents the potential-induced difference spectrum of cyt $c$ between the fully oxidized $(+0.15 \mathrm{~V}$, positive peaks) and the fully reduced $(-0.10 \mathrm{~V}$, negative peaks) state. The spectral features are essentially the same as the previously published result. ${ }^{54}$ The dashed curve represents the potentialinduced difference spectrum of the MPA-modified surface in the absence of cyt $c$. As the potential is raised, a negative band at $1690 \mathrm{~cm}^{-1}$ develops with two positive bands at 1573 and $1388 \mathrm{~cm}^{-1}$. The negative band at $1690 \mathrm{~cm}^{-1}$ is assigned to the $\mathrm{C}=\mathrm{O}$ stretching mode of carboxyl group of protonated MPA. This assignment is substantiated by the concomitant occurrence of the asymmetric (at $1573 \mathrm{~cm}^{-1}$ ) and symmetric carboxylate $\left(\mathrm{COO}^{-}\right)$stretching modes (at $1388 \mathrm{~cm}^{-1}$ ) of deprotonated
MPA. ${ }^{59}$ The appearance of these difference bands indicates that the MPA headgroup deprotonates as the electrode potential increases. ${ }^{60}$

The redox-induced IR difference spectrum of cyt $c$ (continuous trace in Figure 4) shows several bands distinct from the rather broad bands of MPA. The most prominent is a negative band at $1693 \mathrm{~cm}^{-1}$. This band has been assigned to the amide I mode of a $\beta$-turn segment of the reduced form of cyt $c{ }^{49}$ Although the peak position of this band overlaps with the $\mathrm{C}=$ O stretching mode of MPA, the differential amide I bandwidth is much sharper and thus can be easily distinguished. The relative contributions of bands from MPA and from cyt $c$ can also be distinguished from their different potential dependencies (see Figure S4a in Supporting Information). Cyt $c$ bands appear already at $-0.04 \mathrm{~V}$ concomitant with the redox current, while MPA bands dominate at voltages higher than $+0.1 \mathrm{~V}$ (see Figure S4b in Supporting Information).

The complex folding of a protein modulates the amide I vibration of the protein backbone, and several IR bands appear in this energy range. The aforementioned band at $1693 \mathrm{~cm}^{-1}$ is the most intense band, but additional amide I bands are observed at 1666,1646 , and $1627 \mathrm{~cm}^{-1}$ for the reduced state (negative peaks) and at 1672,1659 , and $1634 \mathrm{~cm}^{-1}$ for the oxidized state (positive peaks), respectively. These bands are assigned to different types of structural changes of the protein backbone. A detailed discussion of the assignment of these bands will be presented below.

Potential-Induced IR Difference Spectra of Cytochrome c Adsorbed to a Hydroxyl-Terminated SAM. Figure 5 shows a series of difference spectra at various potentials of cyt $c$ adsorbed to the surface of a self-assembled monolayer of mercaptoethanol (ME SAM). The ME SAM itself does not show any vibrational changes in the observed potential range. However, decreases in the intensity of broad water bands at around 1640 and $3400 \mathrm{~cm}^{-1}$ are observed as the potential increases (data not shown). This potential-dependent change indicates the rearrangement of those water molecules that are hydrogen-bonded to the $-\mathrm{OH}$ terminus of ME. However, the hydration change to the cyt $c$ adsorbed layer is far from being trivial. The surface water that hydrates the ME SAM is replaced by cyt $c$ during the adsorption process. This is detected by the fairly broad decrease in absorbance of the $\mathrm{H}-\mathrm{O}-\mathrm{H}$ bending mode of water around $1640 \mathrm{~cm}^{-1}$ and of the $\mathrm{O}-\mathrm{H}$ stretching vibration at around $3400 \mathrm{~cm}^{-1}$ upon the adsorption of cyt $c$ (see Figure 2). This indicates that the water molecules that hydrate the $-\mathrm{OH}$ group of $\mathrm{ME}$ are replaced by the protein molecules. On this basis, we presume that the difference spectra depicted in Figure 5 solely reflect the potential-induced IR differences of the cyt $c$ layer without any overlapping bands from the ME underlayer.

The potential-induced difference spectrum of cyt $c$ on ME (trace $c$ in Figure 8) significantly differs from that of cyt $c$ on MPA (trace $b$ in Figure 8), although the absolute IR spectra and the electrochemical response are identical (see Figures S2 and S3 and Table S1 in Supporting Information). The most

(58) Yamamoto, H.; Liu, H. Y.; Waldeck, D. H. Chem. Commun. 2001, 10321033.

(59) Colthup, N. B.; Daly, L. H.; Wiberley, S. E. Introduction to Infrared and Raman Spectroscopy; Academic Press: 1990.

(60) Fawcett, W. R.; Fedurco, M.; Kovacova, Z. Langmuir 1994, 10, 24032408 . 


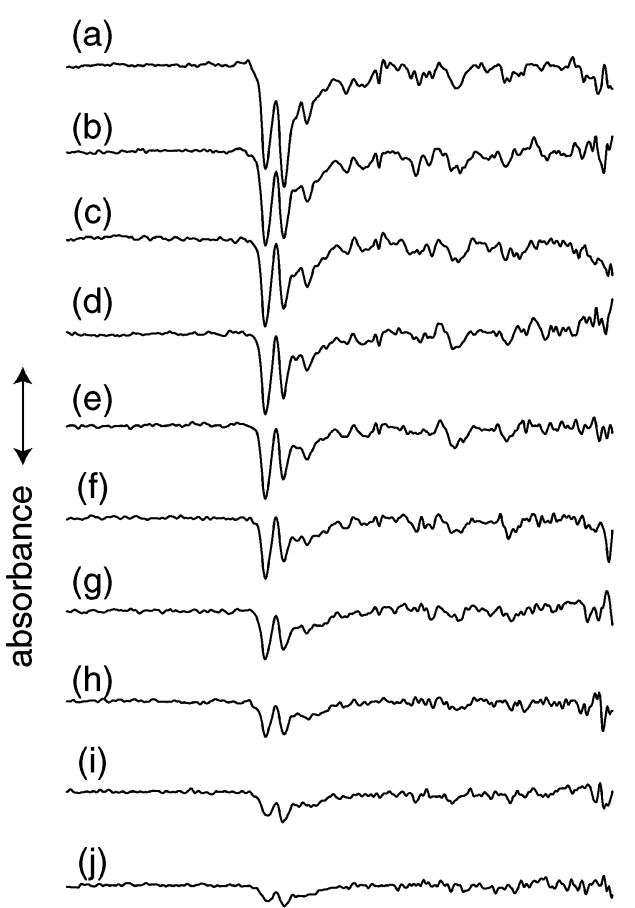

(k)

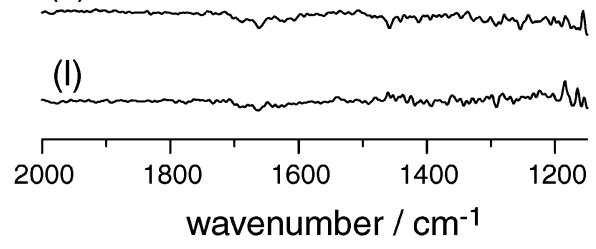

Figure 5. Series of potential-induced IR difference spectra of a cyt $c$ monolayer on mercaptoethanol SAM. Difference spectra have been recorded at voltages of (a) +0.20 , (b) +0.15 , (c) +0.12 , (d) +0.10 , (e) +0.08 , (f) +0.06 , (g) +0.04 , (h) +0.02 , (i) 0.00 , (j) -0.02 , (k) -0.04 , and (l) -0.06 $\mathrm{V}$ with the reduced state as reference $(-0.10 \mathrm{~V})$. All potentials are referred to the $\mathrm{Ag} / \mathrm{AgCl}$ electrode. The arrow corresponds to an absorbance of $\Delta A$ $=5 \times 10^{-4}$.

prominent difference is the intensification of the negative bands at 1662 and $1627 \mathrm{~cm}^{-1}$ relative to the $1693 \mathrm{~cm}^{-1}$ band. Another distinct feature is that the band intensities of the oxidized state are much weaker than those of the reduced state. It might be argued that the latter observation is due to an irreversible desorption of cyt $c$ upon oxidation. This can be refuted, however, by the fact that the redox charge observed by cyclic voltammetry is completely reversible. The decrease of the relative intensities in the oxidized state is rather explained by the near-field effect of SEIDAS ${ }^{50}$ and the movement of cyt $c$ away from the electrode upon oxidation (vide infra).

Despite the differences in the relative intensities of observed difference bands of cyt $c$ between ME and MPA SAM, their peak positions are almost identical. The change in band intensity reflects the different quantities of the change in protein structure. These changes are dependent on the orientation and the distance of cyt $c$ from the surface, both of which are critically influenced by the interaction with the SAM layer. The frequency of the band, however, is indicative for the structural changes of the active site and its immediate environment that take place between the reduced and oxidized states. The identical vibrational frequencies in the spectra of cyt $c$ adsorbed to MPA and ME (Figures $8 \mathrm{a}$ and $8 \mathrm{~b}$ ) provide evidence that the internal

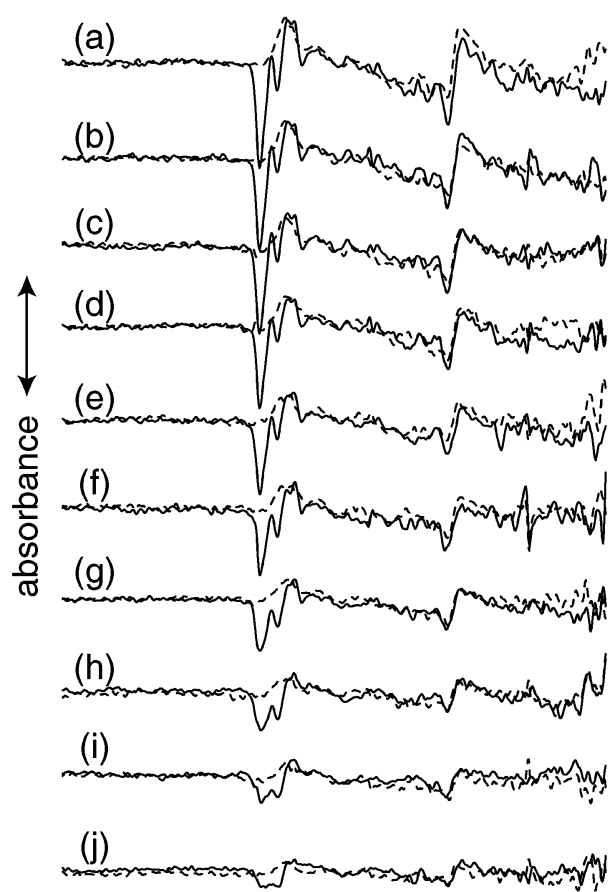

(k)

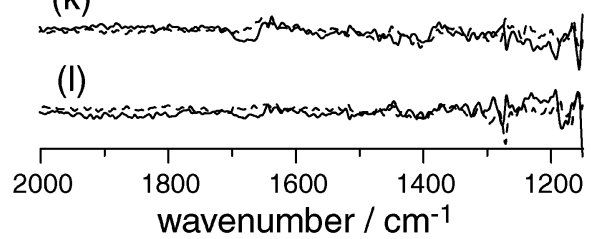

Figure 6. Set of potential-induced IR difference spectra of a cyt $c$ monolayer adsorbed on a cysteine SAM between the fully reduced state $(-0.10 \mathrm{~V})$ and various oxidation potentials: (a) +0.20 , (b) +0.15 , (c) +0.12 , (d) +0.10 , (e) +0.08 , (f) +0.06 , (g) +0.04 , (h) +0.02 , (i) 0.00 , (j) -0.02 , (k) -0.04 , (1) $-0.06 \mathrm{~V}$ vs $\mathrm{Ag} / \mathrm{AgCl}$ (solid curve). The dashed curves represent the potential-induced difference spectra of Cys SAM in the absence of cytochrome $c$. The arrow corresponds to an absorbance of $\Delta \mathrm{A}=5 \times$ $10^{-4}$.

conformational changes of cyt $c$ being triggered by the electron transfer are not influenced by the interaction with the SAM layer.

It has been suggested that the redox reaction between cyt $c$ and an $-\mathrm{OH}$-terminated SAM is entirely diffusion controlled and that cyt $c$ does not adsorb to that surface. ${ }^{7}$ However, this suggestion is inconsistent with our results. Due to the shortrange effect of SEIRA, the observed vibrational spectra arise solely from that part of the monolayer that directly interacts with the ME SAM layer. In addition, the slow adsorption kinetics on the $-\mathrm{OH}$-terminated surface suggests that the surface-bound cyt $c$ is under the influence of the adhesive force from the surface.

Potential-Induced IR Difference Spectra of Cytochrome c on a Cysteine SAM. Figure 6 shows a series of potentialinduced IR difference spectra of cyt $c$ adsorbed to a monolayer of cysteine. Although it is not as apparent as with DTDP or with MPA SAM, Cys SAM also contributes slight potentialinduced spectral changes to the redox-induced difference spectra of cyt $c$. This is demonstrated by the potential-induced difference spectra of the Cys SAM in the absence of cyt $c$ (dashed traces in Figure 6). It is obvious that the broad peak at $1652 \mathrm{~cm}^{-1}$ and a differential-shaped band feature at 1396/1379 $\mathrm{cm}^{-1}$ are the potential difference bands contributed by the Cys layer. The former band is assigned to the asymmetric $\mathrm{NH}_{3}{ }^{+}$bending mode 


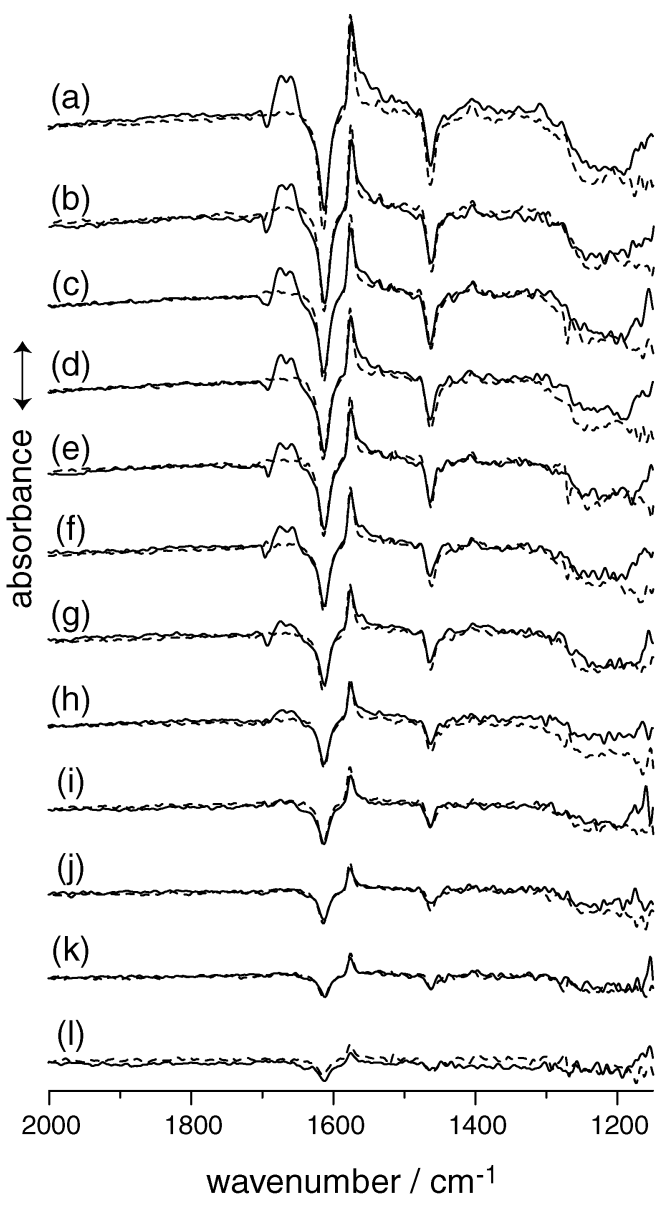

Figure 7. Set of potential-induced IR difference spectra of a cyt $c$ monolayer adsorbed on a DTDP SAM between the fully reduced state $(-0.10 \mathrm{~V})$ and various oxidation potentials: (a) +0.20 , (b) +0.15 , (c) +0.12 , (d) +0.10 , (e) +0.08 , (f) +0.06 , (g) +0.04 , (h) +0.02 , (i) 0.00 , (j) -0.02 , (k) -0.04 , (l) $-0.06 \mathrm{~V}$ vs $\mathrm{Ag} / \mathrm{AgCl}$ (solid curve). The dashed curves represent the potential-induced difference spectra of DTDP SAM in the absence of cytochrome $c$. The arrow corresponds to an absorbance of $\Delta A=5 \times 10^{-4}$.

of cysteine, while the latter is due to the $\mathrm{CH}_{2}$ bending mode. ${ }^{61,62}$ Because these contributions are fairly broad, they are easily distinguished from the cyt $c$ difference bands. The subtraction of the cysteine SAM bands yields the pure response of cyt $c$ when adsorbed to the cysteine SAM (spectrum d in Figure 8). The peak positions closely agree with those observed for cyt $c$ adsorbed to a SAM of MPA (spectrum b in Figure 8) and ME (spectrum $\mathrm{c}$ ). The strong negative bands of cyt $c$ at 1663 and $1691 \mathrm{~cm}^{-1}$ vary in relative intensity when adsorbed to the three different SAMs (Figure 8, spectra $\mathrm{b}-\mathrm{d}$ ). Overall, the redoxinduced IR difference spectrum of cyt $c$ adsorbed to the Cys SAM is more similar to that of cyt $c$ adsorbed to the ME SAM rather than to the MPA SAM. This resemblance between Cys and $\mathrm{ME}$, despite the differences in $1645-25 \mathrm{~cm}^{-1}$ region, indicates the presence of a similar surface conformation of cyt $c$ on both SAMs. This corresponds to the finding that the redox kinetics of cyt $c$ on Cys is similar to that on a ME SAM. ${ }^{1}$

Potential-Induced IR Difference Spectra of Cytochrome $c$ on a 4,4'-Dithiodipyridine SAM. The IR differences of cyt $c$ adsorbed to a DTDP SAM (Figure 7) are very distinct from

(61) Susi, H.; Byler, D. M.; Gerasimowicz, W. V. J. Mol. Struct. 1983, 102, 63-79.

(62) Ihs, A.; Liedberg, B. J. Coll. Int. Sci. 1991, 144, 282-292.

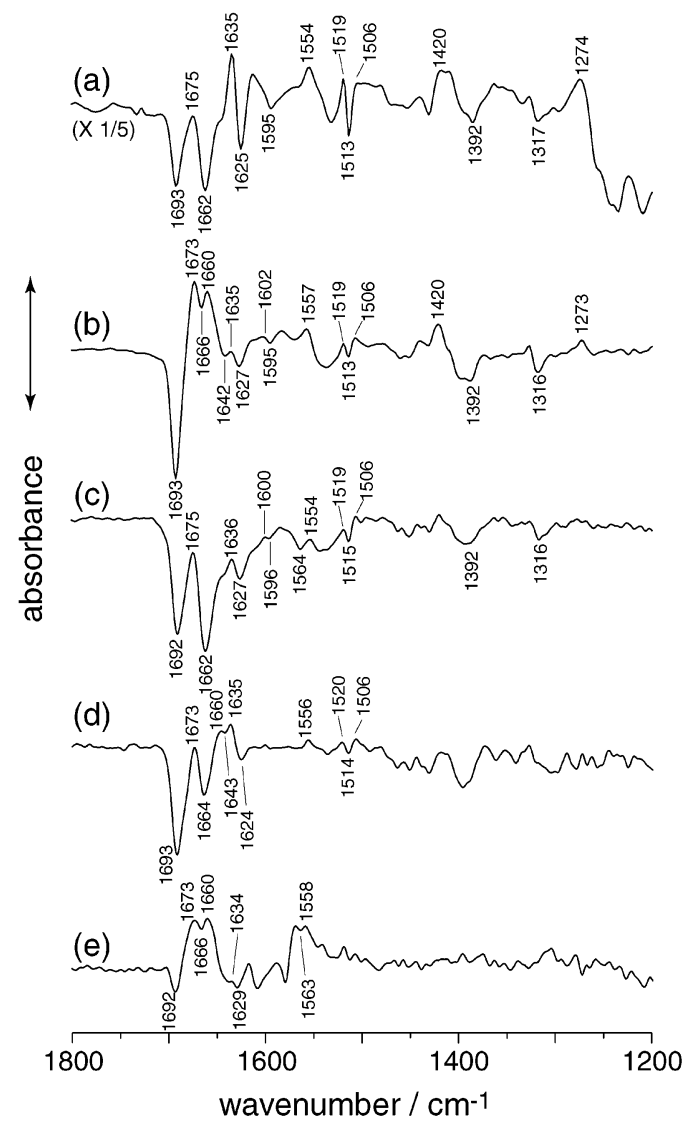

Figure 8. Redox-induced IR difference spectra of cytochrome $c$ in solution (a) as compared to the spectra of a cytochrome $c$ monolayer adsorbed on various CMEs: (b) MPA, (c) ME, (d) Cys, and (e) DTDP. The response by the surface modifier layer has been subtracted to recover the spectra of the adsorbed cyt $\mathrm{c}$. The arrow corresponds to an absorbance of $\Delta A=5 \times$ $10^{-4}$.

those of the SAMs presented above (Figures 4-6 and $8 b-d$ ). The dashed lines represent IR difference spectra under otherwise identical conditions but without cyt $c$ adsorption. Two negative peaks at 1620 and $1466 \mathrm{~cm}^{-1}$ are assigned at $v_{8 \mathrm{a}}$ and $v_{19 \mathrm{a}}$ modes of the protonated pyridine ring vibration, respectively, whereas the positive peak at $1576 \mathrm{~cm}^{-1}$ is assigned to the $v_{8 \mathrm{a}}$ mode. ${ }^{63,64}$ The assignment of these spectral changes is in agreement with the deprotonation of the pyridine group of DTDP as the potential increases. ${ }^{65-67}$ Although the $\mathrm{p} K_{\mathrm{a}}$ of bulk pyridine is at 8.83 , the acidity of pyridine increases when assembled to a monolayer. The $\mathrm{p} K_{\mathrm{a}}$ shifts to 6.06 at $-0.04 \mathrm{~V}$ (vs NHE) and to 3.14 at $+0.54 \mathrm{~V}$ (vs NHE). ${ }^{65-67}$ Thus, in the potential range of our experiment ( -0.1 to $0.2 \mathrm{~V}$, Figure 7 ), the effect of the potentialinduced deprotonation directly influences the structure of the DTDP SAM.

Because the potential-induced difference bands of DTDP are relatively strong, they obscure the cyt $c$ bands in the 1620 to $1570 \mathrm{~cm}^{-1}$ region. Nevertheless, subtraction of the spectral response of the DTDP layer reveals the fine features of the adsorbed cyt $c$ (Figure 8, spectrum e). The amide I bands of the reduced cyt $c$ are observed at 1692, 1666, and $1629 \mathrm{~cm}^{-1}$, while those of the oxidized states are at 1673, 1660, and 1634

(63) Kline, C. H.; Turkevich, J. J. Chem. Phys. 1944, 12, 300

(64) Corrsin, L.; Fax, B. J.; Lord, R. C. J. Chem. Phys. 1953, 21, 1170-1176.

(65) Bryant, M. A.; Crooks, R. M. Langmuir 1993, 9, 385-387.

(66) Smith, C. P.; White, H. S. Langmuir 1993, 9, 1-3.

(67) Wan, L. J.; Hara, Y.; Noda, H.; Osawa, M. J. Phys. Chem. B 1998, 102, 5943-5946. 
$\mathrm{cm}^{-1}$. Although the region between 1620 and $1570 \mathrm{~cm}^{-1}$ is still obscured, a fine structure is revealed at $1563 / 1558 \mathrm{~cm}^{-1}$ that is attributed to the amide II mode. Most strikingly, the observed frequencies of the cyt $c$ difference spectrum adsorbed to DTDP correspond to those from the other SAMs (spectra $b-d$ in Figure 8). However, an increase in intensity of the amide I bands is noted in the oxidized state relative to those of the reduced state. The intensity of the amide I bands at 1692 and $1666 \mathrm{~cm}^{-1}$ in the reduced state, which are the strongest bands with the other SAMs used, are much weaker than the corresponding oxidizedstate bands at 1673 and $1660 \mathrm{~cm}^{-1}$.

Contrary to the other SAMs employed, the redox-induced IR differences, when cyt $c$ is adsorbed to the DTDP SAM, gradually decrease at prolonged measuring times. The peak height of the cyt $c$ band at $1671 \mathrm{~cm}^{-1}$ decreases to about $35 \%$ after $200 \mathrm{~min}$. The bands of DTDP also decrease in intensity concomitantly with the decrease of the cyt $c$ bands. We conclude that the instability of the DTDP layer is induced by the presence of cyt $c$ since the bare DTDP layer is sufficiently stable prior to the addition of cyt $c$. This result indicates that both the adsorbed cyt $c$ and DTDP layer is denatured during cycling of the potential. It has been suggested ${ }^{68}$ that the addition of pyridine induces a structural change in the heme binding pocket of cyt $c$ replacing the heme-ligating Met-80 directly or to either Lys group around heme or pyridine itself. The decrease in the intensity of the DTDP spectrum indicates the detachment of the pyridine moiety from the SAM layer. These detached pyridine molecules may play a role in the denaturation of the adsorbed cyt $c$ either by direct ligation to the heme or by the replacement of the Lys ligand. ${ }^{68}$

\section{Discussion}

Our results demonstrate that cyt $c$ adsorbs to surface modifiers of a different chemical nature. Recording of redox-induced difference spectra of only a monolayer of cyt $c$ adsorbed to those surface modifiers was successfully demonstrated by exploiting the surface-enhancement exerted by the modified gold surface. Moreover, we were able to show that the relative band intensities of the potential-induced difference spectra of cyt $c$ depend critically on the type of surface modifiers. This suggests that there are different types of interaction between cyt $c$ and the various modifiers that determine the presence of different surface structures of cyt $c$ with respect to, e.g., orientation, binding site, etc. The correspondence of the peak position in the redoxinduced difference spectrum of cyt $c$ when adsorbed to the different modifier surfaces or even when dissolved in solution ${ }^{48}$ suggests that the internal conformational change of cyt $c$ triggered by the electron transfer is not influenced by the interaction with the modifier.

On the basis of these findings, we attempt to elaborate a detailed model of the surface structure of cyt $c$ that is derived from the following line of arguments: (I) We revisited the previous band assignment of the IR difference spectrum of cyt $c$ because former studies have pointed out that their assignment does not fully agree with the results from NMR spectroscopy ${ }^{35-38}$ or X-ray crystallography. ${ }^{39-41}$ Our band assignment is supposed to resolve these inconsistencies. (II) On the basis of the band assignment, we will determine the corresponding amino acid

(68) Fan, C. H.; Gillespie, B.; Wang, G. M.; Heeger, A. J.; Plaxco, K. W. J. Phys. Chem. B 2002, 106, 11375-11383. residues that are involved in the molecular changes of cyt $c$ induced by the redox process. (III) Finally, the surface structure of the cyt $c$ monolayer can be derived accounting for several characteristics of SEIDA and results from recent researches.

Assignment of the Observed IR Difference Bands. The bands appearing in the region between 1700 and $1600 \mathrm{~cm}^{-1}$ are all assigned to the amide I mode of the peptide backbone, which is mainly composed of the $\mathrm{C}=\mathrm{O}$ stretching mode. ${ }^{29}$ The peaks are at $1693,1662-6,1642$, and $1627 \mathrm{~cm}^{-1}$ for the reduced state and at 1673,1660 , and $1635 \mathrm{~cm}^{-1}$ for the oxidized state, in good agreement with the results obtained from bulk cyt $c$ (see Figure 8, trace a, and the literature ${ }^{34,49}$ ). The most prominent peak in the difference spectrum is the negative band at 1693 $\mathrm{cm}^{-1}$. We assign this band to the amide I mode of a type III $\beta$-turn according to the experimental and theoretical assignment performed by Krim et al. ${ }^{29}$ These authors suggested that type I and type III $\beta$-turn structures exhibit a band near $1690 \mathrm{~cm}^{-1}$. We prefer the type III assignment because cyt $c$ does not form a type I turn according to the X-ray results. ${ }^{39-41} \mathrm{Krimm}$ et al. also pointed out that care has to be taken not to interpret this band as being due to a characteristic mode of the antiparallel pleated $\beta$-sheet structure. Indeed, Calvert et al. ${ }^{69}$ have assigned this band to the high-frequency component of an extended $\beta$-stranded structure that occurs paired with a low-frequency band at $1634 \mathrm{~cm}^{-1}$. They suggested that a new $\beta$-sheet structure is formed during reduction of cyt $c$. However, this conclusion has been confirmed neither by X-ray crystallography ${ }^{39-41}$ nor by NMR spectroscopy. ${ }^{35-38}$ Calvert et al. ${ }^{69}$ claimed that this discrepancy is due to the conformational averaging factors in these techniques because X-ray crystallography and NMR spectroscopy require long data collection times and cannot follow the substantial dynamic nature of the conformational changes of cyt $c$. In our IR difference spectra, we could not find the corresponding low-frequency component at $1634 \mathrm{~cm}^{-1}$, but a weak band appears at $1628 \mathrm{~cm}^{-1}$. The extinction coefficient of the high-frequency component of the amide I of a $\beta$-strand is much smaller than that of the low-frequency component. $^{31-33}$ Thus, the interpretation by Calvert et al. ${ }^{69}$ apparently contradicts this observation. It should be noted that the surface selection rule of SEIRA ${ }^{50}$ might alter the relative intensity between the 1693 and $1628 \mathrm{~cm}^{-1}$ bands. Yet, the 1693 $\mathrm{cm}^{-1}$ band in the difference spectrum of bulk cyt $c^{48}$ shows much stronger intensity than that of $1628 \mathrm{~cm}^{-1}$, which also refutes the $\beta$-strand assignment. Considering the fact that the secondary structure of cyt $c$ is mainly composed of $\alpha$-helices (47 residues) and turn structures (6 turns) while the $\beta$-strands found in the oxidized state comprises only three interstrand main chain hydrogen bonds, ${ }^{41}$ it is more plausible to assign the band at $1693 \mathrm{~cm}^{-1}$ to a change in $\beta$-turn structure.

It has been suggested that large frequency shifts result when the dihedral angles of a given $\beta$-turn are only slightly varied. ${ }^{29}$ Along this line, we assign the positive band at $1672 \mathrm{~cm}^{-1}$ to a type III $\beta$-turn, which represents the counterpart of the negative band at $1693 \mathrm{~cm}^{-1}$. Since the turn structure often takes the role of a pivot for the adjacent secondary structures, it is plausible that the dihedral angles in these turn structures are readily influenced by the conformational change of the protein. As a consequence for the redox-induced difference spectrum, the peak

(69) Calvert, J. F.; Hill, J. L.; Dong, A. Arch. Biochem. Biophys. 1997, 346, 287-293. 
Table 1. Vibrational Assignment of the Observed IR Bands of Cytochrome $c$

\begin{tabular}{|c|c|c|c|c|}
\hline $\begin{array}{l}\text { reduced } \\
\text { state }\end{array}$ & $\begin{array}{l}\text { oxidized } \\
\text { state }\end{array}$ & assignment & designation & residues \\
\hline 1693 & & amide I & $\beta$-turn type III & $14-19,67-70$ \\
\hline & 1673 & amide I & $\beta$-turn type III & \\
\hline 1662 & 1660 & $\begin{array}{l}\text { amide I } \\
\text { amide I }\end{array}$ & $\begin{array}{l}\beta \text {-turn type II } \\
\beta \text {-turn type II } \\
\text { and/or } \alpha \text {-helix }\end{array}$ & $32-35,35-38$ \\
\hline 1642 & & amide I & (unordered) & \\
\hline 1627 & 1635 & $\begin{array}{l}\text { amide I } \\
\text { amide I }\end{array}$ & $\begin{array}{l}\text { extended } \beta \text {-strand } \\
\text { extended } \beta \text {-strand }\end{array}$ & $37-40,57-79$ \\
\hline $\begin{array}{l}1595 \\
1564\end{array}$ & 1602 & $\begin{array}{l}\text { heme } v_{37} \\
\text { heme } v_{37} \\
\text { amide II }\end{array}$ & $\begin{array}{l}v\left(\mathrm{C}_{\beta} \mathrm{C}_{\beta}\right) \\
v\left(\mathrm{C}_{\beta} \mathrm{C}_{\beta}\right) \\
\beta \text {-turn type III }\end{array}$ & \\
\hline & 1556 & amide II & $\beta$-turn type III & \\
\hline 1514 & 1519 & $\begin{array}{l}\text { tyrosine } \\
\text { tyrosine }\end{array}$ & $\begin{array}{l}\text { in-plane ring vibration } \\
\text { in-plane ring vibration }\end{array}$ & 48,67 \\
\hline & 1506 & tyrosine & in-plane ring vibration & \\
\hline & 1420 & $\mathrm{CH}_{2}, \mathrm{CH}_{3}$ & $\mathrm{C}-\mathrm{H}$ bending & \\
\hline $\begin{array}{l}1392 \\
1316\end{array}$ & 1273 & $\begin{array}{l}\text { amide III } \\
\text { amide III }\end{array}$ & ( $\beta$-turn type III) & \\
\hline
\end{tabular}

position of the amide I mode shifts from $1693 \mathrm{~cm}^{-1}$ in the reduced state to $1672 \mathrm{~cm}^{-1}$ in the oxidized state and, thus, provides a derivative-like spectral feature with a rather narrow bandwidth.

On the same basis, we assign the band at 1666 and 1660 $\mathrm{cm}^{-1}$ to type II $\beta$-turn structure of the reduced and oxidized states, respectively. The type II $\beta$-turn mode is predicted to occur between 1666 and $1656 \mathrm{~cm}^{-1} .^{29}$ The latter band is close to a strong IR band of the $\alpha$-helix at $1658 \mathrm{~cm}^{-1}$ (Table 1). Thus, it is also possible that the amide I mode from the $\alpha$-helix contributes to the $1660 \mathrm{~cm}^{-1}$ band.

The negative band at $1642 \mathrm{~cm}^{-1}$ is assigned to that part of the protein backbone that adopts a random conformation in the reduced state. The pair of difference bands at $1635 / 1627 \mathrm{~cm}^{-1}$ is assigned to the low-frequency component of the $\beta$-strand structure in the oxidized/reduced states, respectively. We presume that the corresponding high-frequency components are covered by the strong difference band from the amide I of the $\beta$-turn at $1693 \mathrm{~cm}^{-1}$ (see above). Although horse heart cyt $c$ contains only a small portion of $\beta$-sheet residues, they are directly hydrogen bonded to the heme propionates, which undergo a major displacement during the redox reaction.

It should be noted that the possible occurrence of difference bands due to heme vibrations has not yet been included in our discussion, although the heme moiety is the central player in the redox process. Most of the heme vibrational bands of cyt $c$ have been assigned by resonance Raman spectroscopy, ${ }^{24,25,70,71}$ including several bands in the $1700-1600 \mathrm{~cm}^{-1}$ region. However, because of the $D_{4 h}$ symmetry of the porphyrin ring system, the extinction rule applies to these modes where Raman modes are inactive in the IR. Although symmetry breaking occurs due to the side chain residues and the distortion of the heme plane, Spiro and co-workers ${ }^{70,71}$ concluded that the intensity of the IR bands (classified as $\mathrm{E}_{\mathrm{u}}$ mode for in-plane vibration) is much smaller as compared to the Raman modes (classified as $\mathrm{A}_{1 \mathrm{~g}}, \mathrm{~B}_{1 \mathrm{~g}}$, and $\mathrm{B}_{2 \mathrm{~g}}$ modes). The only band that might be a candidate for an IR active heme mode is a very small

(70) Li, X. Y.; Czernuszewicz, R. S.; Kincaid, J. R.; Stein, P.; Spiro, T. G. J. Phys. Chem. 1990, 94, 47-61.

(71) Hu, S. Z.; Spiro, T. G. Biophys. J. 1993, 64, A157. derivative-shaped feature at 1602/1595 $\mathrm{cm}^{-1}$, hardly distinguishable from the noise level. It can be assigned to the $v_{37}$ stretching vibration of the heme moiety that is supposed to absorb at around $1600 \mathrm{~cm}^{-1} \cdot 72,73$

Most of the bands in the spectral region between 1580 and $1520 \mathrm{~cm}^{-1}$ can be assigned to amide II vibrations. In general, the amide II band is much less amenable to secondary structure analysis. In fact, the broad feature at $1535 \mathrm{~cm}^{-1}$ (Figure 8) may result from the strong overlap of several amide II bands, which makes their assignment very difficult. Nevertheless, we tentatively assign the derivative feature at $1564 / 1556 \mathrm{~cm}^{-1}$ as a $\beta$-turn on the account of its distinct appearance in the amide I region (at $1693 \mathrm{~cm}^{-1}$ ). Though small in size, the derivative-shaped feature at $1519 / 1514 / 1506 \mathrm{~cm}^{-1}$ is apparent in the difference spectra $b-d$ in Figure 8. It agrees well with a band feature observed with cyt $c$ in solution (spectrum a in Figure 8). From the insensitivity to deuteration, Schlereth et al. ${ }^{49}$ could distinguish this band from an amide II mode and assigned it to the in-plane ring vibration of a tyrosine residue.

Up to now, we have neglected spectral contributions from vibrations of the side chains of the amino acids. These contributions are regarded to be minor because the redox process of cyt $c$ is accompanied by only small conformational changes. There are no indications for protonation/deprotonation or other chemical reactions of the side chains ${ }^{15,16}$ that would lead to stronger difference contributions in the amide range. ${ }^{74,75}$

Correlation with Tertiary Structure. In the previous section, we performed the band assignment that will be now correlated to the tertiary structure of cyt $c$. Recent X-ray and NMR studies ${ }^{35-41}$ suggest that cyt $c$ does not reveal large conformational changes when going from the oxidized to the reduced state. There appear to be rather slight adjustments in the vicinity of the heme environment in response to the electrontransfer reaction. Takano et al. ${ }^{40}$ suggested that the conformation of the porphyrin ring changes between the redox states. It appears to be flatter in the oxidized state, while the bond length between the heme iron and the six ligand atoms are maintained. This leads to changes in the bond angle of the two axial ligands, His-18 and Met-80, and also shifts the position of the propionate groups at the heme end. As a consequence, the hydrogenbonding pattern of the environment is rearranged and shifts the related residues as result. These comprise residues Tyr-67, Thr78, Asn-52, and an internal water molecule that form $\mathrm{H}$-bonds with Met-80; residues Thr-19 and Lys-27 to His-33 for formation of H-bonds with His-18; and Arg-38, Tyr-48, Thr49, Trp-59, and Thr-78 for H-bonding to the propionate group of heme. Shifts of these residues' positions obviously involve a rearrangement of the protein backbone. It has also been suggested that this rearrangement increases the exposure of the heme moiety to the outer solvent when it changes from the reduced state to the oxidized state. ${ }^{39,40}$ The positional shifts of the amino acid residues during the redox reaction are summarized in Figure 9.

Among those, residues 14-19 and 67-70 comprise a type III $\beta$-turn. Thus, the band at $1693 \mathrm{~cm}^{-1}$, which indicates the

(72) Abe, M.; Kitagawa, T.; Kyogoku, Y. J. Chem. Phys. 1978, 69, 45264534 .

(73) Kitagawa, T.; Abe, M.; Ogoshi, H. J. Chem. Phys. 1978, 69, 4516-4525.

(74) Venyaminov, S. Y.; Kalnin, N. N. Biopolymers 1990, 30, 1243-1257.

(75) Rahmelow, K.; Hübner, W.; Ackermann, T. Anal. Biochem. 1998, 257, $1-11$. 


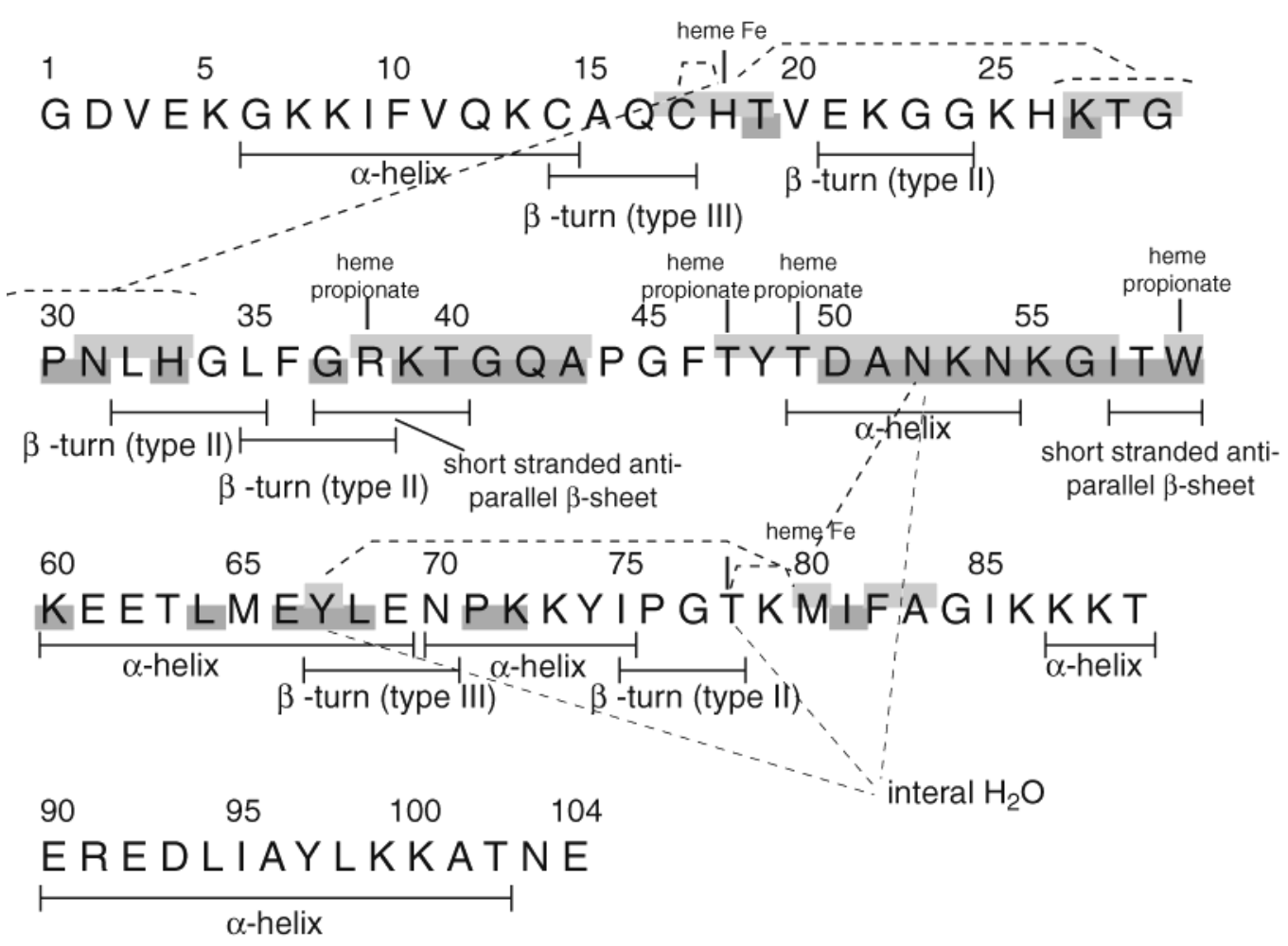

Figure 9. Schematic drawing of the secondary structure of horse heart cytochrome $c$. The shaded area represents the amino acid residues that exhibit a spatial shift during the redox process as detected by X-ray crystallography (light gray shaded, taken from the literature, ${ }^{41}$ PDB entry: $1 \mathrm{HRC}$ ) and NMR spectroscopy (lower part, taken from the literature, ${ }^{35} \mathrm{PDB}$ entries: 1OCD and 2FRC). Broken lines indicate hydrogen-bonding interactions.

presence of a type III $\beta$-turn, can be assigned to the backbone of either of these residues (or to both). Type II $\beta$-turns are found at residues $32-35$ and at $35-38$. The former residues are involved in hydrogen-bonding to His-18, while the latter are hydrogen-bonded to the one of the heme propionates. Thus, the band at around $1661 \mathrm{~cm}^{-1}$ is assigned to the amide I mode of the backbone of these residues. The short antiparallel $\beta$-sheet structure belongs to residues $37-40$ and $57-79$. Both include residues that are involved in hydrogen-bonding to the heme propionate at residues 38 and 59. Therefore, an amide I band can be expected in this frequency range and, accordingly, the difference band pair at $1635 / 1627 \mathrm{~cm}^{-1}$ is assigned to a change in the backbone motions of this $\beta$-sheet. The remainder of the residues may belong to the band at $1642 \mathrm{~cm}^{-1}$, which has been assigned to random structures. Vibrational signatures of changes in the $\alpha$-helical parts of the protein are more difficult to assess. It might well be that the helices (residues 50-55, 60-66, and 71-74, see Figure 9) move as rigid bodies with no changes in the intrahelical H-bonds. Nevertheless, the frequency of the band at $1660 \mathrm{~cm}^{-1}$ is typical for a polypeptide in $\alpha$-helical conformation.

Besides these amide I bands, only the band feature at 1519/ $1514 / 1506 \mathrm{~cm}^{-1}$ has been assigned to an amino acid side chain vibration, namely, to the phenyl ring vibration of a tyrosine. ${ }^{48,57}$ Out of the 4 tyrosines of horse heart cyt $c$, only two are involved in the redox-induced conformational change (Tyr-48 and Tyr67, Figure 9). ${ }^{39,40}$ Thus, the tyrosine band is assigned to either or both of these residues. It is important to note that Tyr-67 exhibits one of the largest positional shifts during the redox reaction of cyt $c$. The phenolic oxygen of Tyr-67 is hydrogenbonded to the sulfur of Met-80 being a ligand of the heme iron. The change in charge of the heme iron causes a rearrangement of hydrogen-bond, giving rise to the change in the phenyl ring vibration of Tyr-67.

Adsorption Site of Cytochrome $c$ and Its Surface Orientation. It has been pointed out that the redox-induced difference spectrum of cyt $c$ in bulk solution is invariant to the chemical nature of the used surface modifiers. ${ }^{49}$ The same investigation demonstrated that the influence of $\mathrm{pH}$ and temperature significantly depends on the type of the modifiers, which has been attributed to a different interaction between cyt $c$ and the modifier molecules. In our experiments where we selectively observe those cyt $c$ molecules that are bound to the surface, the spectra exhibit distinct differences in the relative band intensities being critically dependent on the type of surface modifier. This seeming contradiction between bulk and surface spectra of cyt $c$ can be resolved by considering the relationship between the surface structure of cyt $c$ and the characteristics of SEIRA, namely, (i) the surface selection rule and (ii) the optical nearfield effect. ${ }^{50,76}$ The former effect implies that only those vibrations are IR-active whose dipole moment changes perpendicular to the (local) surface. The latter effect involves that the enhancement of the IR signal is strongest at close proximity to the surface $(<8 \mathrm{~nm})$ and decays faster the further away the molecule is. If cyt $c$ adopts different orientations along the surface, these peculiarities of SEIRA will lead to major alterations in the intensities of the vibrational modes of those residues that change the direction of the dipole moment and that are close to the surface. As a proof of principle, the intensity of the Tyr band at $1519 / 1513 / 1506 \mathrm{~cm}^{-1}$ relative to the amide I band at $1693 \mathrm{~cm}^{-1}$ is much smaller in the SEIDA spectra of the cyt $c$ monolayer (Figure $8 \mathrm{~b}-\mathrm{e}$ ) than that of cyt $c$ in the

(76) Johnson, E.; Aroca, R. J. Phys. Chem. 1995, 99, 9325-9330. 
(a)

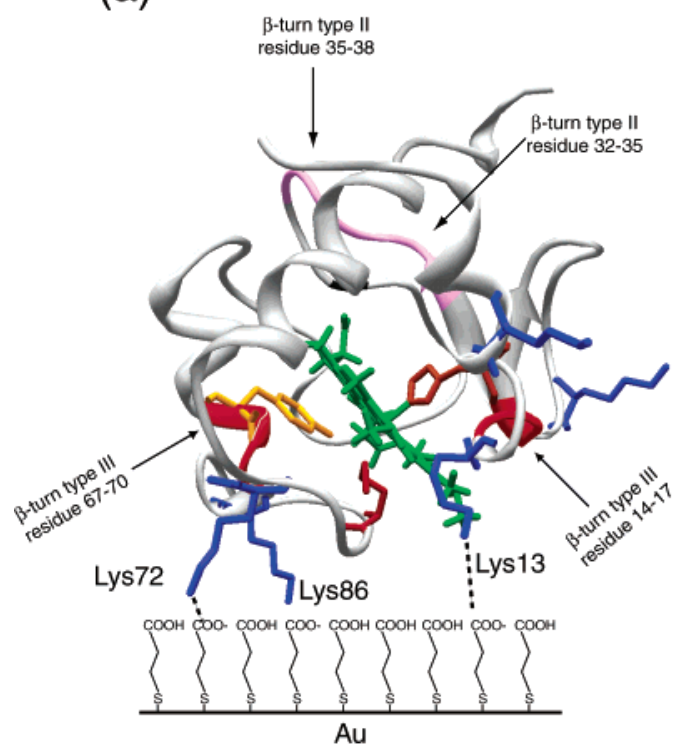

(b)

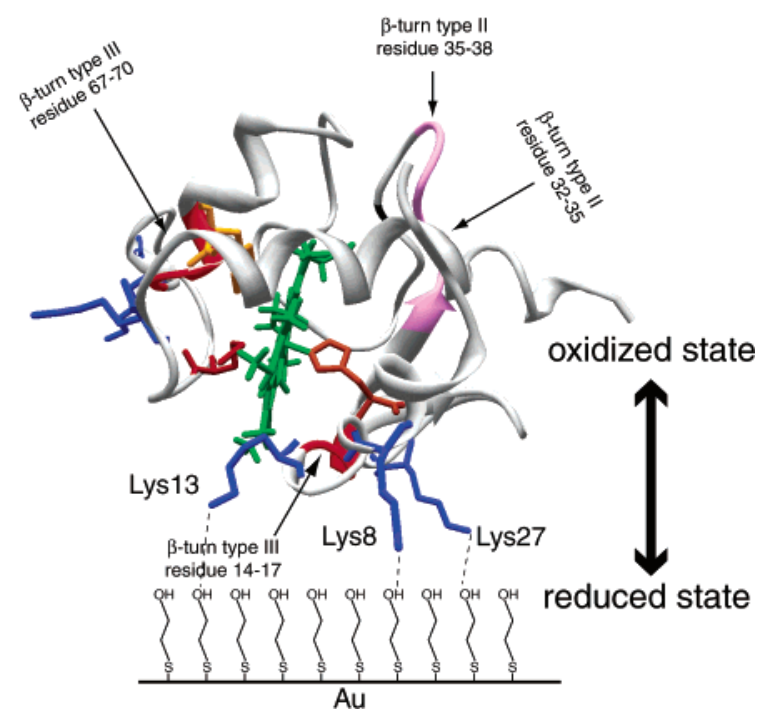

Figure 10. Surface adsorption models of cytochrome $c$ derived from our SEIDAS results. Those Lys residues that are involved in the adsorption to the self-assembled monolayer (SAM) are colored in blue. (a) Cyt $c$ adsorbed on a SAM from mercaptopropionic acid (MPA) via strong interaction of the terminal amine groups of Lys-13, 72, and 86, respectively, with the carboxylate surface of MPA (thick broken line). (b) Orientation of cyt $c$ on mercaptoethanol (ME) where only weak interaction is exerted through Lys-8, 13, and 27 (thin broken line). The arrow on the right side indicates the direction of positional shift of the entire protein upon oxidation (up) and reduction (down). The colored parts of the protein backbone (red for residues 14-17, pink for residues $32-35$ and 35-38, red for residues 67-70) represent $\beta$-turn structures that undergo a conformational change during the redox process as assigned by IR spectroscopy. The cyt $c$ structure was created by Swiss PDB viewer 3.7 with crystallographic data taken from the protein data bank (PDB entry: 1HRC).

bulk (Figure 8a). Because this band is assigned to the in-plane ring-breathing mode of the phenyl ring, it is concluded that the aromatic ring plane of the Tyr is oriented away from the surface normal. As another example, the intensity of the type II $\beta$-turn band at $1662 \mathrm{~cm}^{-1}$ relative to $1693 \mathrm{~cm}^{-1}$ of the type III $\beta$-turn is stronger for cyt $c$ on the ME and Cys SAM, while they are very weak on the MPA and DTDP SAM. Since the type III $\beta$-turn band provides the strongest intensity, it can be presumed that the (backbones of) residues $14-19$ or $67-70$ are located close to the surface. If cyt $c$ orients such that the type II $\beta$-turns (comprising residues 32-35 and 57-79) are closer to the surface, they provide a cyt $c$ difference spectrum as measured in the presence of ME or Cys SAM surface.

The dependence of the orientation of the heme cofactor has been studied by total internal reflection fluorescence spectroscopy when cyt $c$ is adsorbed to substrates with various chemical properties. ${ }^{15}$ It has been concluded that the heme plane is tilted by $46^{\circ}$ with respect to the surface normal with a narrow angular distribution $\left( \pm 6^{\circ}\right)$ when adsorbed to carboxyl-terminated molecules. The heme orients at an angle of $13^{\circ}$ (with respect to the surface normal) on hydrophilic or hydroxy SAM-coated glass, albeit with a broad angular distribution $\left( \pm 30^{\circ}\right)$. A similar orientation has also been concluded from surface-enhanced resonance Raman (SERRS) spectroscopy, although a definite orientation angle has not been specified..$^{20,21}$ These results reveal relevance with recent reports that the strength of the molecular interaction between SAM and cyt $c$ that carboxyl- or pyridineterminated surface provide relatively stronger adsorption, while hydroxyl group or cysteine provide weakest.

The determination of the heme orientation still leaves the orientation of the whole cyt $c$ molecule ambiguous. However, it is possible to deduce the orientation of cyt $c$ by considering the active binding site to its cognate binding partner such as cytochrome $c$ oxidase and cytochrome $b c l$. Cyt $c$ is highly polarized due to the anisotropic distribution of positively charged residues (Lys, Arg) along the protein surface. They form a patch that plays a major role in the interaction with the binding partner prior to electron transfer. It has been suggested that Lys-8, 13, $27,72,79$, and 86 are involved in the binding, while the other lysine residues are not important for ET activity. ${ }^{77,78}$ These results suggest that the orifice of the heme crevice needs to be oriented toward the ET partner for optimal electron exchange.

On the basis of these findings, we propose the following model for the surface structure of cyt $c$ adsorbed on the carboxyl-terminated SAM (Figure 10a). In this orientation, the type III $\beta$-turns comprising residues $67-70$ and $14-17$ locate relatively closer to the electrode surface than the type II $\beta$-turn motif of the residues $32-35$, as indicated by the stronger intensity of the $1693 \mathrm{~cm}^{-1}$ band as compared to the $1662 \mathrm{~cm}^{-1}$ band. Among the 19 lysines of horse heart cyt $c$, Lys-72 and 86 are closest to the SAM surface in this orientation. Thus, these Lys residues comprise the dominant interaction site of cyt $c$ with the SAM surface. It should be noted, however, that in a recent report by Niki et al. ${ }^{14}$ the nearby Lys-13 is favored as a major interaction site but the role of Lys-72 is challenged. We cannot resolve this issue here but rather include the terminal amine groups of lysines 13, 72, and 86 as the dominant electrostatic interaction site with the carboxyl moiety of MPA. It is less plausible to postulate an alternative model where Lys27 and 8 are closest to the surface without breaking the heme orientational condition. In such a model, the type II $\beta$-turns would be closer to the surface than the type III $\beta$-turn moieties. This would lead to a more intense band at $1662 \mathrm{~cm}^{-1}$ as compared to the $1693 \mathrm{~cm}^{-1}$ band, which is contrary to our observation (Figure 8).

(77) Rieder, R.; Bosshard, H. R. J. Biol. Chem. 1980, 255, 4732-4739.

(78) Smith, H. T.; Staudenmayer, N.; Millett, F. Biochemistry 1977, 16, 49714974. 
Figure $10 \mathrm{~b}$ depicts the model of cyt $c$ adsorption on an $\mathrm{OH}$ terminated SAM surface with the heme angle being close to perpendicular to the surface. In this orientation, the type III $\beta$-turn of the residues 67-70 are farther away from the surface then in the case of the MPA surface (Figure 10a), whereas the type II $\beta$-turns moved slightly closer toward the surface. The type III residues $14-17$ have not changed their relative distance to the SAM surface as compared to the carboxylterminated SAM (Figure 10a). This orientation implies a stronger intensity of the $1662 \mathrm{~cm}^{-1}$ band relative to that at $1693 \mathrm{~cm}^{-1}$. Although this model requires that Lys 8, 13, and 27 approach the SAM surface, these residues do not necessarily act as specific binding sites to the surface. The broad angular distribution of the heme orientation and the weak interaction with cyt $c$ on the $-\mathrm{OH}$-terminated SAM suggest that some of the surface cyt $c$ may be adsorbed with different orientation by the involvement of the other Lys residues, namely, Lys 72 and 86. Thus, this model rather provides an average orientation, while the model for carboxyl SAM comprises a definite orientation of cyt $c$.

The adsorption structures of cyt $c$ on Cys and DTDP SAM are unclear due to the lack of information on the heme orientation. For the Cys SAM, however, a similar adsorption structure as compared to the $-\mathrm{OH}$-terminated SAM is expected judging from the similarity of the potential-induced difference spectra (Figure 8, spectra $\mathrm{c}$ and $\mathrm{d}$ ). It has been suggested that the Cys SAM layer forms an electrostatic adsorbate-adsorbate interaction within the monolayer ${ }^{79-81}$ through their carboxyl and amine headgroups. Such cysteine-cysteine interactions can compensate for the negative charges of the carboxyl moiety in cysteine and compete with the interaction of the Lys residues of cyt $c$ with the SAM layer. This leads to a weak interaction between cyt $c$ and cysteine SAM, and provides a similar surface structure to $-\mathrm{OH}$-terminated SAM.

The adsorption structure of cyt $c$ on DTDP SAM is expected to be different from the other SAMs, considering its particular spectral features (Figure 8e). It is possible that the pyridine moiety may interact with cyt $c$ in a completely different manner than lysine binding sites. Recently, Waldeck and co-workers have reported on the attachment of cyt $c$ to a pyridine-terminated long-chain alkanethiol with pyridine acting as a ligand of the heme iron. ${ }^{58,82,83}$ It is well-known that the addition of pyridine induces a conformational change of cyt $c$ by influencing the heme ligation. ${ }^{68}$ Although DTDP does not possess a sufficiently long alkane chain, the pyridine moiety may tend to form an interaction directly with the heme iron. This may explain the instability of DTDP adsorbed cyt $c$ during the redox-induced experiments. The adsorption of cyt $c$ destabilizes the DTDP layer by retracting the pyridine moiety. This may cause the detachment of the pyridine moiety during the long time measurement. The detached pyridine from the SAM layer will ligate to the

(79) Fawcett, W. R.; Fedurco, M.; Kovacova, Z.; Borkowska, Z. Langmuir 1994, 10, 912-919.

(80) Fawcett, W. R.; Fedurco, M.; Kovacova, Z.; Borkowska, Z. J. Electroanal. Chem. 1994, 368, 265-274.

(81) Fawcett, W. R.; Fedurco, M.; Kovacova, Z.; Borkowska, Z. J. Electroanal. Chem. 1994, 368, 275-280

(82) Wei, J. J.; Liu, H. Y.; Dick, A. R.; Yamamoto, H.; He, Y. F.; Waldeck, D. H. J. Am. Chem. Soc. 2002, 124, 9591-9599.

(83) Wei, J. J.; Liu, H. Y.; Khoshtariya, D. E.; Yamamoto, H.; Dick, A.; Waldeck, D. H. Angew. Chem., Int. Ed. 2002, 41, 4700-4703. heme iron of the adsorbed cyt $c$, which leads to the denaturation of the protein.

It is interesting to note that the amide band of cyt $c$ on carboxyl-terminated SAM shows equal intensities on both the reduced and the oxidized states. In the case of the $-\mathrm{OH}-$ terminated or Cys SAM, the intensity of amide I band in the oxidized state is weaker than that of reduced state. This may be explained by the shift of the relative distance of cyt $c$ from the electrode surface. Since cyt $c$ is firmly bound to the carboxylterminated SAM, it is expected that the relative position of the protein from the surface is fixed during the redox process. In the case of the $-\mathrm{OH}$ and the cysteine-terminated SAM surface, the oxidized cyt $c$ may be further away from the surface than the reduced cyt $c$ due to the weak interaction. This provides a weaker IR signal in the oxidized state across the entire spectral range as experimentally observed (Figure 8, spectra $\mathrm{c}$ and d). The opposite applies to the pyridine-terminated SAM where the intensities of the amide bands are stronger in the oxidized state than in the reduced state. This suggests that cyt $c$ is located closer to the electrode surface in the oxidized state than in the reduced state. This implies that the surface of a pyridineterminated SAM exerts a different type of interaction with the adsorbed cyt $c$.

\section{Conclusion}

In this work, we have measured potential-induced difference IR spectra of the electron-transfer reaction taking place between a monolayer of cyt $c$ and a Au electrode modified by carboxyl, hydroxyl, zwitterionic, and pyridine-terminated SAMs. Although the frequencies of the vibrational bands are identical for the studied surface modifiers, the relative peak intensities strongly depend on the type of surface modifier. The former observation suggests that the functionality of cyt $c$ is fully preserved independent of the surface modification, while the latter observation is attributed to the differences in surface structure, i.e., the orientation and the relative distance of cyt $c$ from the surface. Integrating the vibrational analysis with results from NMR spectroscopy and X-ray crystallography provides details on the involvement of specific amino acids in the conformational changes during the redox process. By comparison with results on the heme orientation and the active binding site of cyt $c$, the adsorption structures of cyt $c$ monolayer are derived from our IR results. In these models, cyt $c$ adsorbs on carboxyl-terminated SAM through Lys-72, Lys-86, and (probably) Lys-13. This provides a surface orientation in which the amino acid residues $32-38$ (type II $\beta$-turn segment) are more distant from the electrode surface than residues $67-70$ (type III $\beta$-turn segment). The equal intensity of the oxidized and reduced amide bands suggests that cyt $c$ is fixed at a certain distance from the electrode surface during the redox process. The adsorption of cyt $c$ to hydroxyl-terminated or to cysteine SAMs is mainly performed by Lys-8, 13, and 27 . In this orientation, residues $32-38$ are located closer to the electrode surface relative to residues $67-70$. The asymmetric feature of the amide band intensity in which the reduced state appears stronger than the oxidized state suggests that the distance of cyt $c$ from surface is increased when the protein is oxidized. We conclude from the relative intensity between the oxidized and the reduced states in the cyt $c$ 
difference spectrum that cyt $c$ adsorbs to a pyridine-terminated SAM in an entirely different manner than with the other SAMs.

Acknowledgment. We acknowledge Prof. G. Büldt for continuous generous support. This work has been supported by a grant from the Volkswagen Foundation ("Intra- und Intermolekulare Elektronenübertragung"). We are grateful to the
Alexander-von-Humboldt foundation for providing a fellowship to K.A.

Supporting Information Available: Figures S1-4 and Table S1 (PDF). This material is available free of charge via the Internet at http://pubs.acs.org.

JA048346N 